\title{
MODELO ESTRUCTURAL DE ENFERMERIA DE CALIDAD DE VIDA E INCERTIDUMBRE FRENTE A LA ENFERMEDAD
}

\author{
QUALITY OF LIFE AND UNCERTATINTY TOWARDS DISEASE: \\ A NURSING STRUCTURAL MODEL
}

ALEXANDRA TORRES A.* y OLIVIA SANHUEZA A.**

\begin{abstract}
RESUMEN
Se presenta un modelo estructural que relaciona el concepto de calidad de vida y nivel de incertidumbrefrenteal cáncer como enfermedad, a ser aplicado a pacientes con cáncer de mamas, cérvico uterino y de vesícula en el Servicio de Oncología del Hospital Guillermo Grant Benavente del Servicio de Salud Concepción. Su formulación forma parte del desarrollo de una Tesis deD octorado en Enfermería dela U niversidad deConcepción, cuyo propósito es indagar respecto a la influencia del nivel de incertidumbre en la calidad de vida de estas pacientes. Este modelo discute los aspectos conceptuales del constructo calidad devida y nivel de incertidumbre, basado en los modelos de L. Schwartzmann y de M. M ishel, respectivamente, proponiendo una integración dinámica de ambos. El modelo propuesto será valorado empíricamenteen el transcurso del trabajo de la tesis, a objeto deser incorporado en la val oración de las pacientes con diagnóstico de cáncer y posterior intervención de enfermería, destinado a mejorar la calidad de vida de esas mujeres.
\end{abstract}

Palabras claves: Calidad de la vida, nivel de incertidumbre, modelo estructural de valoración eintervención de enfermería.

\begin{abstract}
A nursing structural model that relates quality of life concept and uncertainty level towards cancer as disease is presented; this model is meant to be applied in patients with Breast Cancer, Cervical Cancer, and Gallbladder Cancer, attending Guillermo Grant Benavente H ospital Oncology Unit, from Concepcion Health Service. Its formulation is part of a Nursing Doctoral Thesis from University of Concepcion, aiming to find out about the influence concerning the uncertainty level in quality of life of these patients. This model discuss the conceptual facts of the quality of life construct and uncertainty level, based on L. Schwartzmann and M. M ishel models, as respectively, proposing a dynamic integration of both ones. The proposed model will be empirically assessed during the period the doctoral thesis lasts, in order to be included in patients with cancer diagnosis assessment and nursing intervention, as well, so intended to improve the quality of life in these women
\end{abstract}

Keywords: Quality of life. Uncertainty level, Assessment and Intervention Structural M odel.

Fecha de recepción: 30/03/06. Fecha aceptación: 01/06/06

\section{INTRODUCCION}

Los cambios en la estructura social y económica ocurridos durante los últimos 100 años han tenido un efecto fundamental en la salud dela población, modificando sustancialmente el perfil epidemiológico y las condiciones de la salud y enfermedad (Gordon, 1998). Las personas tienen una mayor esperanza devida, prevalecen las enfermedades degenerativas lo

\footnotetext{
*Enfermera. Licenciada en Epidemiología, Magister en Bioestadística Universidad de Chile. Candidato a Doctor Programa D octorado en Enfermería, Universidad de Concepción, Concepción. E-mail: atorres@ssconcepcion.cl

** Doctora en Enfermería. Profesor Titular, Departamento de Enfermería, Facultad de Medicina, Universidad de Concepción, Concepción, Chile. E-mail: osanhue@udec.cl
} 
que trae consigo un significativo impacto en su calidad de vida.

La aparición de este concepto como tal y la preocupación por su evaluación sistemática y científica se inicia al rededor de la década de los 60, cuando la atención de salud es influenciada por los cambios sociales y nuevos modelos epidemiológicos de salud enfermedad. En este nuevo escenario se resta importancia a la cantidad (morbilidad, mortalidad), para favorecer la calidad de vida humana: al iviar síntomas, mejorar el nivel de funcionamiento, conseguir mejores relaciones sociales, autonomía, etc., paradigma que cambia el concepto de cuidados de salud y en donde se empieza a valorar el impacto de las enfermedades y sus tratamientos en función del bienestar y satisfacción de los pacientes, su capacidad física, psíquica y social ( $M$ artínez y Lozano, 1998).

Históricamentehan existido dos constructos básicos del concepto de calidad devida: el primero, considerado como una unidad integral y, el segundo, compuesto por un conjunto deunidades componentes derivados de la estructura social y el medio ambiente general. Felcey Perry (1995) encontraron diversos modelos conceptuales de calidad de vida, añadiendo un cuarto modelo a las tres conceptualizaciones propuestas por BorthwickDuffy en 1992. Según éstas, la calidad de vida ha sido definida como: a) la calidad de las condiciones de vida de una persona; b) como la satisfacción experimentada por la persona con dichascondiciones vitales; c) como la combinación de componentes objetivos y subjetivos, es decir, calidad de vida definida como la calidad de las condiciones de vida deuna persona junto a la satisfacción que ésta experimentay, por último, d) como la combinación de las condiciones de vida y la satisfacción personal ponderadas por la escala de valores, aspiracionesy expectativas personales (Gómez y Sabeh, 2000).

En la actualidad son numerosas las investigaciones dedicadas a la evaluación de la calidad de vida, empleando esteconcepto como un modo de referirsea la percepción que tie ne el paciente de los efectos de una enfermedad determinada, especialmente de las consecuencias que provoca sobre su bienestar físico, emocional y social (Lugones, 2002). Concepto particularmente útil en el estudio de pacientes con patologías crónicas, especialmente las oncológicas, determinados no sólo por las características biológicas de la enfermedad y su connotación psicosocial en la población (Bayes, 1991), sino por el fuerte impacto emocional, físico y social queno sólo afectan al paciente sino también a su entorno familiar (M oro, 1997), y dondela adaptación constituyeun factor significativo para el mantenimiento de un nivel razonable de calidad de vida (Lugo y col., 1998).

Comparativamente, son menos las referencias bibliográficas que vinculan la calidad de vida con la incertidumbre frente a la enfermedad, definido esta última como la incapacidad dedeterminar el significado delos eventos relacionados con la enfermedad y donde la persona que toma la decisión es incapaz de dar val ores definidos a los hechos o no es capaz de predecir qué resultados se obtendrán (M ishel, 1998). Las investigaciones realizadas en este campo demuestran que los indicadores objetivos y subjetivos, tanto de la amenaza de muerte como de la sintomatología de la enfermedad, están sin lugar a dudas relacionados con ambos fenómenos.

Así, con la intención de entender conceptualmente los factores determinantes de la calidad de vida y la incertidumbre que provoca la enfermedad, se han formulado modelos teóricos que tratan de explicar ambos conceptos y cuya aplicación empírica sirvede base para la identificación de estos factores, y la proposición de programas de prevención e intervención.

En concordancia con lo anterior, el propósito de esteartículo esindagar respecto a la influencia de la incertidumbre en la calidad de vida de estas pacientes, mediante la revisión dedos modelos teóricos, cuya aplicación empírica ha sido avalada: el de cal idad de vida 
de L. Schwartzmann (2003) y el de incertidumbre de M. Mishel (1998), proponiendo al mismo tiempo un modelo estructural que décuenta de la relación entre calidad de vida y nivel de incertidumbre y que será aplicado empíricamente a mujeres con cánceres prevalentes en Chile durante las distintas etapas del proceso de su enfermedad.

Para enfermería el conocimiento de la calidad de vida y su asociación con el nivel de incertidumbrefrentea la enfermedad, adquiere gran importancia, lo cual permitiría profundizar en las necesidades de atención de estas pacientes, aportando información a la práctica clínica en el contexto delas enfermedades oncológicas y de los factores psicosociales que en ellas influyen. De este modo, las profesionales de enfermería que tienen a su cargo este tipo de pacientes podrían incorporar estos aspectos a la val oración y la intervención en enfermería en su plan de cuidados; al mismo tiempo elaborar estrategias cuya labor puede extenderse ya sea tanto en la clínica hospitalaria, la atención de enfermería en domicilio, la rehabilitación, la orientación en salud y la consejería familiar para la adaptación a la enfermedad, todo lo cual contribuirá no sólo al mejoramiento de la calidad de vida del paciente y familia, sino al de la propia comunidad.

\section{MODELO DE CALIDAD DE VIDA (SH WATZMAN N, 2003)}

Desde hace dos décadas y producto de las características epidemiológicas y sociales de la enfermedad, dondelas patologías crónicas son prevalentes y para lo cual no existe una curación total, cambia el objetivo delos tratamientos, para ser destinados a atenuar o eliminar síntomas, evitar complicaciones y mejorar el bienestar de los pacientes, utilizándose para ello un modelo alternativo en el cuidado del paciente llamado modelo biopsicosocial, el cual incorpora no sólo el concepto biológico de la enfermedad, sino que adiciona las di- mensiones psicológicas y sociales que experimenta el paciente frentea la enfermedad, surgiendo de esta manera el paradigma de calidad de vida como una medida subjetiva de estos dominios (Boyle, 1997).

Patrick y Erikson (1993) definen la calidad de vida como el valor asignado a la duración de la vida, modificada por las oportunidades sociales, la percepción, el estado funcional que puede ser influenciada por la enfermedad traumatismoso tratamientos. En su medición incluye las funciones físicas, estado psicológico, síntomas somáticos tales como dolor, funciones sociales incluyendo las familiares, funciones sexuales, ocupacionales y posiblemente estado financiero.

Jalowiec (1990) sugirió la existencia de componentes primarios que afectan la calidad de vida y entre los que se incluyen salud, capacidad funcional y la satisfacción por la vida. Padilla y col. (1992) señalaron las cualidades principales dela calidad devida identificándolas como: bienestar psicológico (satisfacción, significado de lavida, logro dela meta y felicidad); bienestar físico (actividades dela vida, del apetito y del sueño diario); bienestar social e interpersonal; y bienestar financiero y material.

M inayo y col. (2000) señalan la relatividad del concepto de calidad de vida, el cual posee tres marcos de referencia: histórico, donde cada sociedad tiene parámetros de calidad de vida en distintos momentos históricos; cultural, dependientes de sus tradiciones culturales, y clase social, donde las expectativas que cada uno tiene están en estrecha relación con los niveles económicos, educacionales y oportunidad de acceso.

La esencia de este concepto es el reconocimiento de que la percepción de las personas sobre su estado de salud, depende en gran medida de sus val ores y creencias, su contexto cultural ehistoria personal, siendo una noción eminentemente humana que se relaciona con el grado de satisfacción que tiene la persona con su situación física, su estado emocional, su vida familiar y social (Schwartzmann, 2003). 
Schwartzmann y col. (1999) proponen un modelo de evaluación de factores psicosociales determinantes de la calidad de vida relacionada con la salud. Este modelo considera que la calidad de vida es un proceso dinámico y cambianteque incluyeinteracciones continuas entreel paciente y su medio ambiente, interacciones determinadas por factores or- gánicos (tipo de enfermedad y evolución), psicológicos (personalidad y grado de cambio en sistema de valores, creencias y expectativas), sociales y familiares (soporte social recibido y percibido), y de cuyas resultantes dependerá el bienestar físico, psíquico y social y en la evaluación general de la vida que hace el paciente (Figura 1).

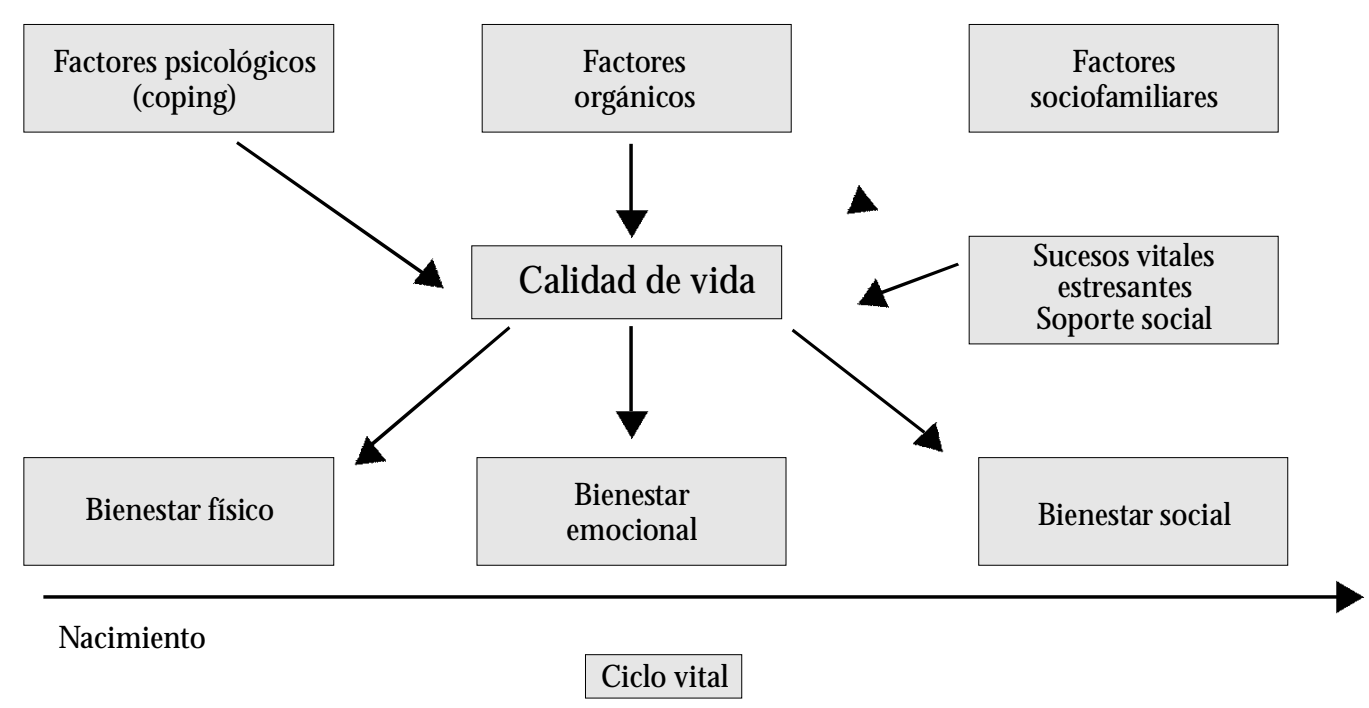

Figura 1. Calidad de vida relacionada con la salud (Schwartzmann, 1999).

Posteriomente, Schwartzmann (2003) presenta un modelo de cal idad de vida adaptado deKuok Fai Leung (Figura2) yen dondepostula el papel de los procesos de adaptación a las nuevas situaciones, los cuales llevan a cambios en la valoración con patrones temporales o interpersonales también cambiantes, lo cual puedeser probado desdeel punto devista clínico a través del estudio delos mecanismos de afrontamiento (coping) y patrones con los cuales la persona se compara.

La calidad de vida resultante dependería de la medida en quelosmecanismos deafrontamiento y la adecuación de las expectativas permitiera que la brecha entre expectativas y percepción dela situación actual no fuera tan disímil. En este mismo sentido actuaría el soporte social percibido, mientras que la acumulación de sucesos vitales que producen estrés, además de la propia enfermedad, incidiría negativamente (pobreza, desempleo, duelos).

Desde la perspectiva de este modelo, para el caso de pacientes con cáncer, el diagnóstico y posterior tratamiento de las enfermedades oncológicas provoca un fuerte impacto emocional, dondeaparecen problemas psicológicos cambiantes, que se suceden en las diversas fases de su enfermedad, fenómeno que dependerá de las estrategias de afrontamiento que utilice para enfrentarse a la enfermedad y que, en suma, determinaran su proceso adaptativo y con ello su calidad de vida (evaluación afectiva y mecanismos de afrontamiento) (Kaufman, 1989).

Los pacientes que están en mejores condiciones para adaptarse podrán continuar con las responsabilidades normales, hacer frente 
a la dificultad física y emocional y seguir participando en actividades que son significativas para ellos, encontrando aún significado e importancia en sus vidas. Los pacientes que no seadapten bien probablementeparticiparán menos en sus propias actividades vitales, se retraerán y perderán esperanza.

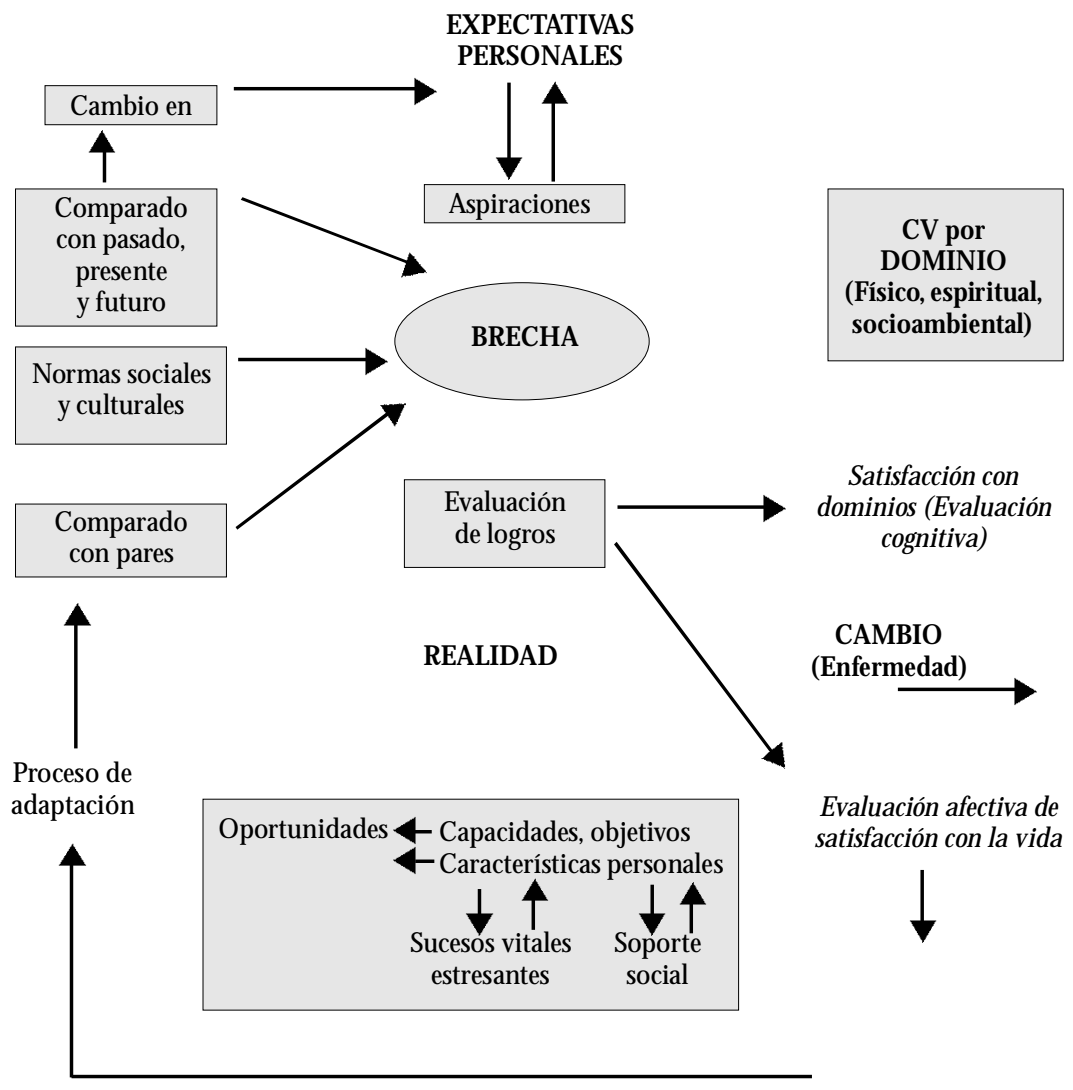

Figura 2. M odelo conceptual de calidad de vida adaptado de Kuok Fai Leung (2002) por Schwartzmann (2003).

\section{MODELO DE INCERTIDUMBRE FREN- TE A LA ENFERMEDAD (MISHEL, 1988)}

M erleM ishel (1988) propuso un modelo para evaluar la incertidumbre frente a la enfermedad basado en modelos ya existentes de procesamiento de la información de Warburton (1979) y en el estudio de la personalidad de Budner (1962).

Mishel atribuye parte de su teoría original al trabajo de Lazarus y Folkman (1984) sobre estrés, valoración afrontamiento; lo que es exclusivo de Mishel es la aplicación del mar- co de la incertidumbre como factor estresante en el contexto de la enfermedad (Bailey y Stewart, 2003). Para ello conceptualiza el nivel de incertidumbre frente a la enfermedad como la incapacidad de la persona de determinar el significado de los hechos relacionados con la enfermedad, siendo incapaz de dar val ores definidos a los objetos o hechos y por lo tanto no puede predecir los resultado, todos, fenómenos motivado por la falta de información y conocimiento (M ishel, 1998).

De esta forma, si las estrategias recopiladas son efectivas, entonces ocurrirá la adap- 
tación. La dificultad de la adaptación dependerá de la habilidad de las estrategias recopiladas para manipular la incertidumbre en la dirección deseada, ya sea para mantenerla, si se valora como oportunidad, o para reproducirla, si sevalora como un peligro (Isla, 1996).

De acuerdo a lo anterior, el modelo de la incertidumbre frente a la enfermedad está basado en los siguientes supuestos:

1) La incertidumbre es un estado cognitivo que representa la inadecuación del esque ma cognitivo existente, cuya función es la de ayudar en la interpretación de los hechos surgidos a raíz de la enfermedad.

2) Laincertidumbreesuna experiencia intrínse camente neutra, queno se desea ni se desprecia hasta que se valora de una u otra forma.

3) La adaptación representala continuidad de la conducta biopsicosocial normal del individuo y constituye un resultado deseado de los esfuerzos del afrontamiento, bien para reducir el nivel deincertidumbre, vista como un peligro, bien para ver la incertidumbre como una oportunidad.

4) La relación entre los casos de enfermedad, la incertidumbre, la valoración, los sistemas de afrontamiento y la adaptación es lineal y unidireccional, y va de las situaciones de incertidumbre a la adaptación.

Elementos conceptuales del modelo:

a) Antecedentes de la incertidumbre: Definidos a través del marco de los estímulos, las capacidades cognitivas y las fuentes de la estructura.

b) Autovaloración de la incertidumbre: Con dos procesos definidos: la inferencia y la ilusión.

c) Afrontamiento: Que tiene como resultado la adaptación. Si la incertidumbre se valora como un peligro, el afrontamiento setraduceen acciones y búsqueda deapoyo psicológico. Si, en cambio, la incertidumbre se ve como una oportunidad, el afrontamiento se protege.

Deacuerdo al modelo, la adaptación siempre ocurre como resultado final del proceso, la diferencia está definida por el factor tiempo y en dondees posible realizar intervenciones específicas dirigidas a minimizar estefactor, lo cual mejorará las estrategias de afrontamiento y determinará el proceso adaptativo mejorando sustancialmentela calidad de vida (Figura 3).

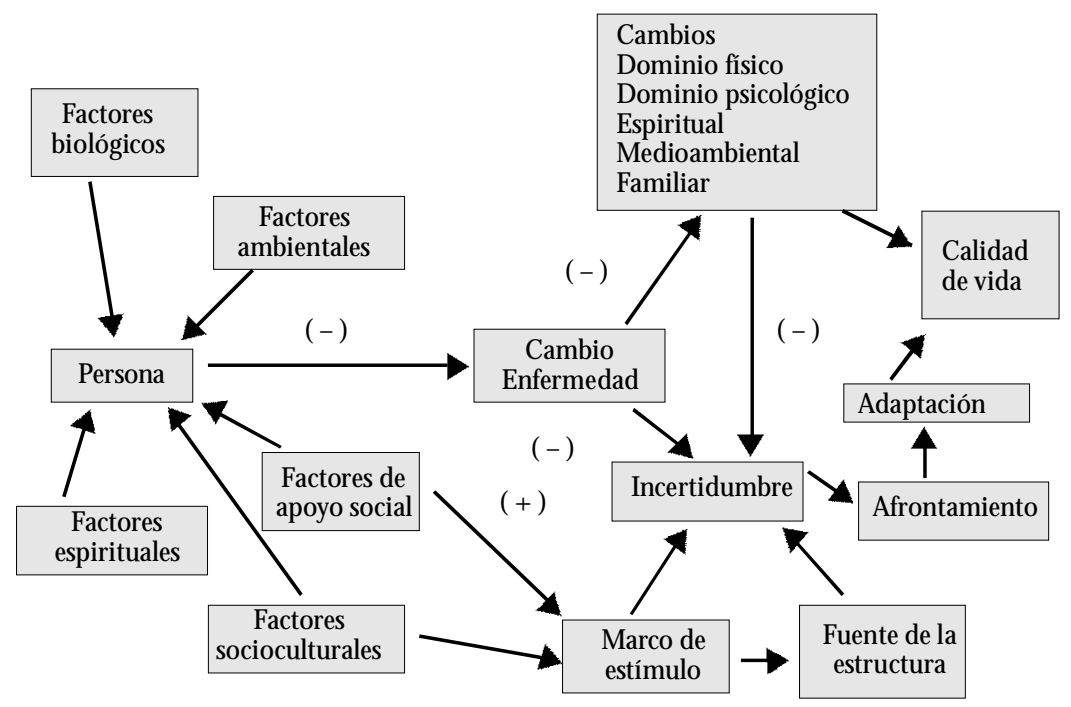

Figura 3. Modelo de incertidumbre percibida frente a la enfermedad (M ishel, 1998). 


\section{MODELO ESTRUCTURAL DE CALIDAD DE VIDA E INCERTIDUMBRE FRENTE A LA ENFERMEDAD}

Teniendo como referencia los modelos expuestos, se proponeun modelo estructural que intenta avanzar en la comprensión de la relación entre la calidad de vida y la incertidumbre frente a la enfermedad. Dicho modelo se fundamenta principalmenteen baseaun modelo de relaciones causales, en las queel cambio en una variable se supone que produce un cambio en otra variable; de este modo las variables son clasificadas en dos clases, las observadas directamente y las latentes, las cuales no son observadas pero están relacionadas con las primeras.

En forma general, el modelo asume que existe una estructuración causal entre las variables latentes y éstas aparecen como causas fundamentales delas variables observadas, las cuales pueden ser analizadas a través dela técnica del modelo de ecuaciones estructurales considerado como una extensión de varias técnicas multivariantes y que tiene la capacidad de analizar relaciones para cada subconjunto de variables (González, 1989).

Para su representación se utiliza un modelo de sendero con las siguientes convenciones en su realización:

1) Las variables observadas se representan en un cuadro, que pueden ser variables exógenas (características propias del paciente: edad, sexo,educación, etc.) o endógenas (características afectadas: calidad de vida, nivel de incertidumbre).

2) Las variables latentes son representadas a través de un círculo o de una elipse.

3) Las flechas unidireccionales indican relaciones causales entre las variables exógenas y endógenas.

El objetivo del modelo es averiguar cómo la incertidumbre afecta a cada uno de los dominios del constructo calidad de vida. La Figura 4 muestra la ilustración del modelo teó- rico estructural propuesto con las variables latentes exógenas y endógenas a analizar.

Así, el modelo establece que la variable latente endógena Calidad de vida se relaciona causalmente con las cuatro variables latentes exógenas: Dominio físico, Dominio psicológico, Dominio social y Dominio del ambiente, y que éstas a su vez se relacionan causalmente con las variables observadas calificadas como P1 a P26 correspondientes a las dimensiones de cada uno de los dominios operacionalizados en la escala de medición abreviada de calidad de vida WHOQOL BREF (Carrasco, 1998).

Por su parte, la variable endógena latente Incertidumbre se encuentra relacionada causalmente con la variable latente endógena Adaptación y ésta con la variable latente endógena Calidad de vida.

A su vez, las variables latentes exógenas M arco de estímulo y Fuentes de la estructura se correlacionan entre ellas y se encuentran relacionadas con las tres variables latentes endógenas Incertidumbre, Adaptación y $\mathrm{Ca}$ lidad de vida.

La variable latente exógena M arco de estímulo es explicada a través de las variables latentes exógenas: Tipología de los síntomas, Coherencia y Familiaridad de los hechos, las tres medidas a través de las variables observadas exógenas que corresponden a las preguntas dela Escala delncertidumbrede M erle Mishel.

Por último, la variablelatenteexógenaFuentes de la estructura es explicada a través de las variables latentes exógenas: Credibilidad en el equipo de salud y Factores biosociodemográficos, medidos a través de las variables observadas exógenas de las preguntas de la Escala de Incertidumbre de $M$ erle $M$ ishel y antecedentes de la paciente.

De este modo, el modelo postula que ante la enfermedad, especial menteaquellas detipo oncológico, seproduce una situación de estrés e incertidumbre, factor que se correlaciona directamente con las características exógenas del paciente y que repercuten significativa- 
mente en su calidad de vida. Estas constituirán un factor condicionante para que el paciente asuma las estrategias de afrontamiento y del tiempo desu adaptación, constituyendo un factor condicionante de su calidad de vida más allá de las características de su propia enfermedad.

La utilidad del modelo reside en dimen- sionar el efecto de cada subconjunto de las variablesanalizadas y tiempos deadaptación, con la finalidad de proponer estrategias de intervención que las enfermeras incluyan en el plan de cuidados del paciente oncológico, permitiendo una mejoría en su calidad devida ante la nueva situación vivencial ocasionada por la enfermedad.

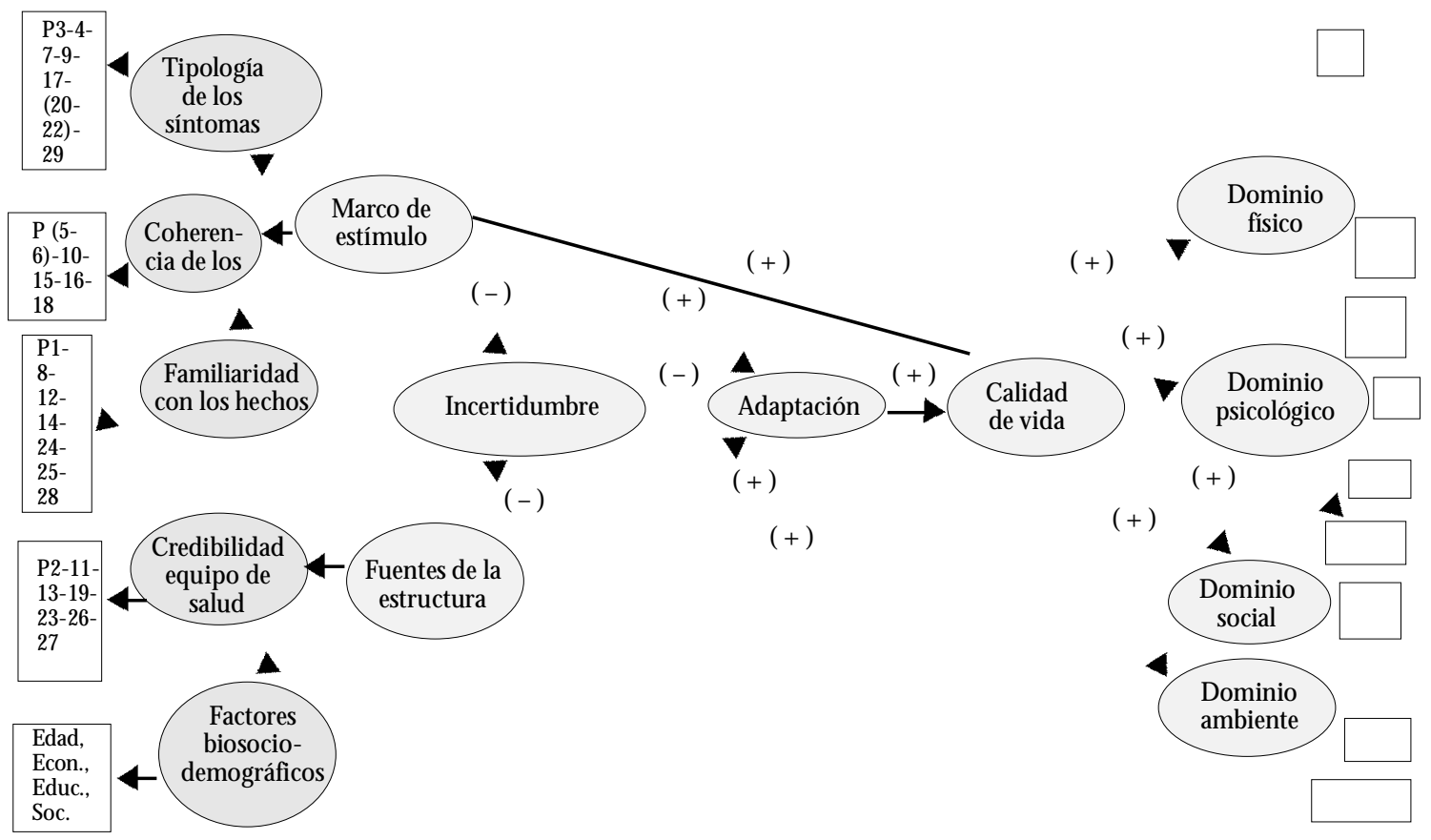

Figura 4. M odelo estructurado calidad de vida e incertidumbre frente a la enfermedad.

\section{CONSIDERACIONES FINALES}

A partir de los modelos teóricos presentados relacionados con la medición de la Calidad de vida y del Nivel de incertidumbrefrentea la enfermedad, se puede concluir que es necesario aportar mucho más conocimiento en este campo, ya sea generando nuevos modelos o la verificación empírica de los mismos, ambos con la finalidad de contribuir a que la atención del paciente y los recursos destinados a los programas de atención, no sólo se fundamente en los aspectos biológicos de la enfermedad (modelo biomédico), sino que tiendan a un mayor desarrollo del modelo integral bio-psicosocial.

Al mismo tiempo, el conocimiento del modelo y la cuantificación del efecto de cada una de las variables componentes de la incertidumbre frente a la enfermedad y su repercusión en la calidad de vida, nos permite otorgar una atención integral, donde la enfermera pueda reconocer en sus pacientes los signos y síntomas críticos quedificulten el afron- 
tamiento y la adaptación en desmedro de la calidad devida, determinando a la vez en qué momentos de la etapa del proceso las intervenciones son más efectivas y oportunas. Proceso quepermitiráayudar a satisfacer no sólo las necesidades integrales de individuo sino de su propia comunidad.

\section{REFERENCIASBIBLIOGRAFICAS}

Bailey, D. Stewart, L. (2003). M erle M ishel. Laincertidumbre frente a la enfermedad. En M arriner, A. Raile, M. (2003). M odel os y teorías en enfermería. (5a ed.). St. Louis: Mosby, 561-582.

Bayes, R. (1991). Psicología oncológica (2a ed). Barcelona: M artínez Roca.

Borthwick-Duffy, S.A. (1992). Quality of life and quality of carein mental retardation. In L. Rowitz (Ed.), Mental retardation in the year 2000 (pp. 52-66). Berlin: Springer-Verlag.

Boyle, C. (1997). M easuring the quality of later life. Rev. Soc. Lond, 352, 1871-1879.

Budner, S. (1962). Intolerance of ambiguity as a personality variable. Journal of personality, 30, 2950.

Carrasco, R.L. (1998). Versión española del Whoqol. Madrid: Ergon.

Felce, D. Perry, J. (1995). Quality of life: It's Definition and Measurement. Research in Developmental Disabilities Vol. 16 (1), 51-74

Gómez, M . y Sabeh, E. (2000). Calidad devida. Evolución del concepto y su influencia en la investigación y la práctica. Instituto Universitario delntegración en la Comunidad, Facultad de Psicología, Universidad de Salamanca. M adrid: Rialp.

González, P. (1989). Aplicación del LISREL al análisis del rendimiento estudiantil. Revista Economía N 4, 55-73.

Gordon, J. (1988). El desafío de la epidemiología. Publicación científica OPS, 500, 10-12.

Isla, X. (1996). Percepción de incertidumbre de los pacientes diabéticos, controlados en el Consultorio Adosado de Especialidades, del Hospital Herminia Martin de Chillán. Chile 1994-1995. Revista Ciencia y Enfermería II (1): 71-80.

Jalowiec, A. (1990) Issues in using multiplemeasures of quality of life. Seminars in Oncology Nursing, 6, 271-277.
Kaufmann, A.E. (1989). Los enfermos frente a su cáncer. En, Kaufmann, A.; Aiach, P.; Waissman, R. La enfermedad grave. Aspectos médicosy psicosociales. M adrid: Editorial Interamericana-Mc Graw Hill.

Lazarus, R.S. Folkman, S. (1984). Stress, appraisal, and coping. New York: Springer Publishing.

Leung, K. F. (2002). A Systemic model for HealthRelated Quality of Life. Newsletter for ISOQOL Menbers 7 (3), 2-3.

Lugo, J. Bacallao, J. Rodríguez, G. (1998). Validez de contenido de un cuestionario para medir calidad de vida en pacientes con cáncer de mamas. Revista Cubana Oncología. Cuba:14 (2), 83-6.

Lugones, M . (2002). Algunas consideraciones sobre la calidad de vida. Revista Cubana M edicina General Integral, 4.

M artínez, V.; Lozano, A. (1998). Calidad de vida de Ios ancianos. Castilla: Colección Ciencia y Técnica. Editorial Universidad de Castilla-La M ancha.

M ishel, M.H. (1998). Uncertainty in illness. Image Journal of Nursing Scholarship, 20, 225-231.

M inayo, M.C.; Hartz, Z.; M ., Buss, P. (2000). Quality of life and Health: a necessary debate. Ciencia \& Saúde Colectiva. ABRASCO 5 (1), 7-18.

Moro, L. (1997). Variables que influyen en la respuesta psicológica al diagnóstico de cáncer. Extraído el 22 de abril 2005, disponible en http:// www.fs-morente.filos.ucm.es/publicaciones/ iberpsicología/iberpsi2/moro/moro.htm

Padilla, G.; Grant, M .; Ferrell, B. (1992). Nursing research into quality of life. Quality of Life Research 1, 341-348.

Patrik, D. \& Erikson P. (1993). Health status and healthy policy: allocating resources to health care. En: Boyle C. (1997). M easuring the quality of later life. Rev. Soc. Lond. B (1997), 352, 18711879.

Schwartzmann, L.; Olaizola, I. Guerra, A. et al. (1999). Validación de un instrumento para medir calidad devida en hemodiálisis crónica: Perfil de impacto de la enfermedad. Revista M édica del Uruguay Vol. 15. Agosto: 103-109.

Schwartzmann, L. (2003). Calidad deVida relacionada con la salud: Aspectos conceptual es. Revista Ciencia y Enfermería IX (2): 9-21.

Warburton, D.M . (1979). Physiological aspects of information processing and stress. In V. Hamilton $\&$ D.M . Warburton (Eds). Human stress and cognition: An information procesing approach (pp. 33-65). New York: John Wiley \& Sons. 